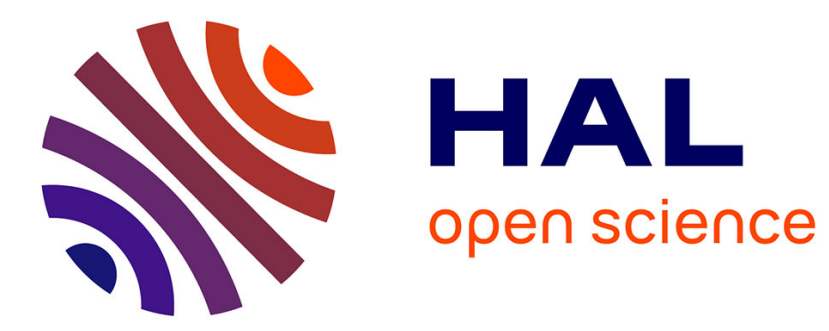

\title{
Troubles dans la communication, recréer la possibilité du dialogue dans les situations de handicap où la fonction du langage est perturbée
}

\author{
Anne-Lyse Chabert
}

\section{To cite this version:}

Anne-Lyse Chabert. Troubles dans la communication, recréer la possibilité du dialogue dans les situations de handicap où la fonction du langage est perturbée. L'esprit créateur, 2021, Disability's Worldmaking: Pasts and Futures, 61 (4), pp.15-31. 10.1353/esp.2021.0044 . hal-03368142

HAL Id: hal-03368142

https://hal.science/hal-03368142

Submitted on 6 Oct 2021

HAL is a multi-disciplinary open access archive for the deposit and dissemination of scientific research documents, whether they are published or not. The documents may come from teaching and research institutions in France or abroad, or from public or private research centers.
L'archive ouverte pluridisciplinaire HAL, est destinée au dépôt et à la diffusion de documents scientifiques de niveau recherche, publiés ou non, émanant des établissements d'enseignement et de recherche français ou étrangers, des laboratoires publics ou privés. 
Anne-Lyse Chabert

\section{Résumé 100 mots}

Mon propre déficit d'élocution m'apprend toujours plus combien avoir du mal à se faire comprendre des autres est une gêne déterminante pour notre vivre ensemble, loin de constituer un simple ajout à mon premier handicap moteur. Ce nouveau handicap semble au contraire le décupler puisqu'il porte atteinte et altère les outils-mêmes qui vont permettre à la personne en situation de vulnérabilité de stabiliser le monde qui l'entoure. Mais une fois l'état de choc dépassé, quelles sont les réinventions que ces personnes peuvent mettre en place pour retrouver cette communication abimée ? En quoi leur milieu peut-il alors les aider?

Troubles dans la communication, recréer la possibilité du dialogue dans les situations de handicap où la fonction du langage est perturbée

«Mais même si les limitations de la communication peuvent provoquer le scepticisme philosophique, la frustration artistique et le silence mystique, le fait surprenant de la communication n'est pas sa limitation, mais sa richesse en dépit de cette limitation. $»^{1}$

Vilem Flusser, «Le phénomène surprenant de la communication »

\section{Introduction}

Mon questionnement prend sa source dans un projet de travail sur l'expérience des personnes en difficulté de communication verbale et sur les réinventions qu'elles mettent en place pour retrouver cette communication perdue ou abimée avec les autres. En effet, mon propre déficit d'élocution ne cesse de m'apprendre combien avoir du mal à se faire comprendre des autres tout autant que mal les comprendre est une gêne cruciale pour notre vivre ensemble, loin de constituer un simple ajout à mon premier handicap moteur : ce nouveau handicap semble au contraire le décupler dans la mesure où il porte atteinte et altère les outils-mêmes qui vont permettre à la 
personne déjà en situation de handicap et donc de vulnérabilité de stabiliser le monde qui l'entoure. Or comme le dit Vilem Flusser : « nous sommes tous profondément engagés en communication, et c'est cet engagement qui donne de la signification à nos vies » (1). Nous avons tous un profond besoin de communiquer, et le plus surprenant dans cette communication toujours maladroite, toujours limitée, n'est pas son caractère frustrant mais le fait qu'elle permette aux uns et aux autres, au cours d'échanges si imparfaits soient-ils, de créer un monde de sens commun.

L'importance du milieu n'est plus seulement accessoire, elle est essentielle dans ce paradigme qui invite également à repenser nos responsabilités : qu'est-ce qui fait qu'un même individu dans deux milieux distincts est alternativement lourdement ou plus du tout handicapé ? Où est le point de bascule qui a permis ce retrait du handicap dans certaines « situations bulle » ? Et comment soigner le handicap au sens de prendre soin, de remettre du « je » dans une situation $a$ priori mal engagée?

J'inscris d'abord mon projet dans le prolongement mais aussi la rupture de mon premier ouvrage. Un second et un troisième temps sont consacrés à des cas particuliers de pathologies du langage à savoir l'aphasie et l'infirmité motrice cérébrale que je décris brièvement sur le plan scientifique avant de m'appuyer sur quelques entretiens ou témoignages. Un dernier axe traite des enjeux philosophiques sur lesquels m’a mené mon travail de réflexion.

\section{Quelles peuvent être les stratégies mises en place ? Quelques enjeux philosophiques}

Mon premier ouvrage Transformer le handicap (paru chez Erès en 2017) ${ }^{2}$, mettait l'accent sur l'inventivité humaine qui peut se déployer dans une situation a priori bouleversée par le handicap. J'ai utilisé pour cela trois concepts que j'ai illustrés chaque fois grâce à une expérience de vie puisque c'est ce que je considère être le cœur de mon problème : le concept de norme à l'échelle du corps, celui d'affordance ou invitation à agir dans le milieu technique, celui de capabilité comme retraçant les frontières normatives 
entre ce que peut être ou ne pas être un individu dans son milieu social. Chacun des trois concepts met au jour l'articulation d'un dialogue retrouvé entre l'individu et son milieu.

Ces trois concepts n'ont toutefois pas la préséance de mon propos à l'image des premières questions de mon auditoire qui semblait fermement s'y attacher ; j'aurais pu utiliser des outils différents pour déployer l'idée qu'améliorer la situation de handicap, c'est une affaire qui passe avant tout au travers de l'expérience vécue du sujet qui se coordonne avec une renégociation de l'ensemble de son milieu. Ici les trois notions que j'ai développées présentent simplement l'avantage de mettre en lumière les trois espaces qui balisent nos vies à tous dans leurs grands traits et prennent en compte ce que peut offrir le milieu en parallèle avec les opportunités que peut saisir l'individu.

Dans quelle mesure ce projet s'inscrit-il également en rupture par rapport à ce travail antérieur tout en le prolongeant ? Je considère en effet que le langage n'est pas un outil comme les autres, mais l'outil par excellence qui nous permet de façonner des médiations (soit d'autres outils) qui nous relient les uns aux autres dans la vie ordinaire, si bien qu'en être privé, c'est ne plus pouvoir accéder librement à ce monde commun. Ce handicap particulier diffère dans sa structure même de la dynamique de transformation que je décris dans mon premier ouvrage, et en exacerbe simplement encore les enjeux. La personne est $a$ priori empêchée non pas seulement de réaliser une activité qu'elle voudrait mettre en œuvre comme le peuvent la plupart de ses congénères, moyennant un détournement en toute intelligence de certaines ressources de son environnement. Elle est bien plutôt empêchée jusque dans la possibilité même d'inciter l'environnement qui l'entoure à s'adapter à la nouvelle configuration qu'elle présente. Au niveau social, ce handicap est mésestimé parce qu'il est assez souvent invisible, pas toujours associé à un handicap moteur ou autre ce qui vulnérabilise d'autant plus les sujets concernés. Ce handicap a priori anodin 
entraîne un immense mal-être de la personne qui n'a pas mis en place de nouvelles façons d'échanger avec le monde qui l'entoure.

J'ai schématisé dans deux diagrammes (schémas 1 et 2) d'une part, cette capacité de l'individu aidé de son environnement à essayer de résoudre plus ou moins inconsciemment les problèmes qu'il affronte, et auxquels nous sommes tous soumis dans la prise de nos décisions quotidiennes : en termes de négociation, de contraintes, de ressources, d'objectifs, d'évaluation, et de création, somme toute la prudence dont parlait déjà Aristote. Dans le second diagramme, un déplacement dans le temps de la place de l'individu qui s'adapte à nos sociétés mais également - ce que nous oublions trop souvent - un élargissement de la société à laquelle il aspire et qui aspire dans le même temps à son accueil, à étoffer ses possibilités d'inclusion afin de le recevoir comme n'importe lequel des individus.

Cette perspective de pensée posée, j'aimerais développer un handicap langagier particulier sur lequel j'ai travaillé, l'aphasie. Après en avoir décrit le spectre nosologique, cause, symptômes, histoire dans les grands traits, je m'intéresse tout particulièrement aux témoignages vécus d'un dessinateur aphasique soigné en 1977 et au point de vue de l'orthophoniste qui l'a alors pris en charge.

Les deux cas d'étude que je développerai par la suite soulèvent des enjeux philosophiques assez différents mais dont les deux principaux gravitent autour de la société inclusive et de sa possibilité, le second enjeu étant de façon assez évidente une vulnérabilité encore accrue lorsqu'on parle de personnes handicapées au niveau de la communication verbale.

Le premier enjeu concerne le monde social qui nous entoure, que d'aucuns voudraient aujourd'hui plus « inclusif », c'est-à-dire invitant à l'inclusion ceux qui sont encore en marge, mais d'une manière ambiguë puisqu'on n'est jamais certain du contenu de 
ce mouvement d'inclusion : va-t-on ramener la norme d'un individu auparavant exclu, ici pour des raisons de handicap, à la norme standard que propose la société, ou va-t-on au contraire lui donner un environnement favorable pour qu'il développe ses propres normes de vie ? En d'autres termes, se situe-t-on dans une logique institutionnelle, ou plutôt dans une logique plus individuelle ? Quelle est la véritable place que veut/peut accorder la société à ces personnes a priori exclues de l'espace public ? Deux processus différents peuvent être mis en place face à l'exclusion a priori par la société standard d'un individu avec un handicap qui peut être celui de la communication orale : soit un mouvement d'extériorité qui consiste à permettre à l'individu de se réaliser, soit un mouvement d'intériorité qui le ramène à une norme plus standard.

On voit très clairement ce qu'on entrapercevait déjà avant, à savoir que l'inclusion de ces personnes handicapées du langage sera encore plus délicate que pour les autres handicaps dans la mesure où cet outil même de la parole n'est pas une possibilité qu'elles ont a priori dans leur bagage pour aménager ou compenser en partie la pesanteur de leur handicap.

D’où l'ambiguïté du travail du thérapeute qui peut facilement déraper et tomber dans un rôle qui consisterait moins à proposer un environnement d'opportunités que le sujet puisse saisir qu'à endosser le rôle de celui qui décide et ne propose plus, en d'autres termes ne fait plus qu'entretenir la dépendance à laquelle est déjà assujettie l'individu au début de sa rééducation. Dans ce paradigme, le soignant n'agirait plus dans l'alliance thérapeutique du patient en acceptant de prendre des risques afin d'aider thérapeutiquement ce dernier, ne laisserait plus sa créativité se déployer librement à partir d'une simple intuition quand il a observé son patient (comme on le verra dans le cas de Philippe Van Eeckhout par rapport à son patient Sabadel). 
Le second enjeu que je développe concerne la vulnérabilité accrue de ce type de public biaisé dans la communication. Ce qu'il faut bien prendre en considération, c'est que nous sommes tous plus ou moins vulnérables et que c'est simplement une situation qui exacerbe ou révèle notre vulnérabilité à un instant donné.

Par ailleurs, même lorsqu'une personne aide et que l'autre est aidée, il y a toujours rencontre de deux vulnérabilités en présence, certes à des niveaux d'exposition plus ou moins intenses, mais une vulnérabilité déjà a priori partagée ; c'est à mon avis un point fondamental pour comprendre l'ensemble de la dynamique de cette situation de handicap mais de beaucoup d'autres aussi, souvent trop peu prises en compte ; comme s'il y avait d'un côté quelqu'un de vulnérable et de façon implicite face à lui, quelqu'un d'invulnérable.

Cette vulnérabilité accrue nous pousse aussi à revisiter les enjeux d'une communication et des difficultés qu'elle peut soulever avant même d'avoir des problèmes d'expression. Au-delà de cette difficulté que nous avons décrite, la communication entre les individus, si elle est bien créatrice de cette espace public, politique, n'est pas exempte d'autres obstacles valables pour tous : communiquer, c'est toujours trouver un terrain commun avec l'autre ce qui implique des efforts mutuels quand je le rencontre, la communication me situe alors dans un espace de négociation où il faut en permanence faire des compromis.

D'autres enjeux touchent au handicap invisible par exemple, facteur d'une plus grande vulnérabilité dans ce contexte. Que veut dire ne pas pouvoir exprimer ses besoins et son avis ? La personne ne risque-t-elle pas d'être surinterprétée à son corps défendant ? Par ailleurs, peut-on penser sans langage ? Dans le cas des personnes aphasiques, comment arriver à réaliser qu'elles n'ont souvent aucun problème de compréhension mais qu'elles ne trouvent pas « le mot pour le dire » ? Un des points également marquants réside dans cet 
effort que l'on fait aujourd'hui pour que tout passe par la médiation d'un outil technologique : rendre accessible la moindre information (en rendant également possible I'action numérique par les mêmes biais). Que penser in fine de cet embryon de projet encore un peu fou (mais pour combien de temps encore ?) de travailler directement sur le matériau de la pensée humaine pour compenser les handicaps à partir de capteurs d'ondes cérébrales?

\section{Premier cas d'étude : les personnes aphasiques}

Le terme aphasie vient du a privatif et de phasein en grec, « dire ", l'aphasie désigne donc étymologiquement « privation de la parole » (aphasique = celui qui n'a pas la fonction de la parole). C'est un trouble du langage dont l'origine vient du système nerveux central. On observe des tableaux cliniques très différents, d'où l'importance d'élaborer une nouvelle thérapie chaque fois et non pas de plaquer des thérapies déjà validées antérieurement sur d'autres patients.

On observe deux grands types d'aphasie, l'aphasie fluente et l'aphasie non-fluente.

- Dans le cas de l'aphasie fluente (ou aphasie de Wernicke), le débit de parole de la personne est incontrôlé et les phrases n'ont pas de cohérence.

- Dans le cas de l’aphasie non-fluente (ou aphasie de Broca), en général la personne comprend tout ce qu'on lui dit mais elle est incapable de retrouver les mots, incapable de nommer les choses.

Le patient aphasique peut espérer une amélioration de son état grâce à l'entraînement et la prise en charge thérapeutique qui doit se faire le plus précocement possible. 
L'expérience d'aphasie vécue sur laquelle je me suis penchée relève d'un double point de vue : le dessinateur Sabadel fait un AVC (accident vasculaire cérébral) en 1977 où il est pris en charge à la Salpêtrière après plusieurs jours d'errance diagnostique par I'orthophoniste Philippe Van Eeckhout qui retrace et décrit le lien qu'il entretient avec ce dernier suite à cet accident dans son livre Le Langage blessé $e^{3}$ Parallèlement, Sabadel nous livre dans son ouvrage Une Plume à mon cerveau ${ }^{4}$ des dessins poignants (dessins 1, 2 et 3), très représentatifs de l'état d'esprit dans lequel il se trouve. Cette vision croisée permet un nouvel éclairage sur une même situation.

Philippe Van Eeckhout décrit son premier contact avec Sabadel dans ces termes : « II pouvait encore émettre quelques sons sans signification mais ne parvenait pas à exécuter des ordres simples comme désigner un verre ou la fenêtre. Il était incapable d'écrire, de se mouvoir. Seul son regard traduisait un désir de communiquer » (3). La démutisation (sortie du mutisme) va donc consister en un déblocage du langage automatique. Le premier mot que Sabadel prononce et répète sans arrêt par la suite (comme le font beaucoup d’aphasiques dans le cadre des stéréotypies au début de la rééducation) sera le mot « bonjour » que ce dernier utilisera alors comme un passe-partout pour répondre à n'importe quelle question (par exemple : il répond « bonjour » à la question « combien avezvous d'enfants ? »). Sabadel éprouve une grande difficulté à ne prononcer que cet unique mot, ce qui le pousse souvent à pleurer avec Van Eeckhout. Ce n'est qu'autour du deuxième mois qu'il parvient à se libérer de cette stéréotypie.

C'est alors que la rééducation prend une nouvelle tournure. Sabadel ne pense qu'à récupérer les fonctions de sa main droite : « vivre pour lui, c'est dessiner » selon Van Eeckhout (3). Van Eeckhout va utiliser cette appétence pour appuyer une nouvelle thérapie autour du langage : il demandera à Sabadel après avoir illustré certaines situations de les 
mettre par la suite en mots sous forme écrite puis orale. « Une fois le dessin achevé, Sabadel devait écrire sous la dictée puis lire à haute voix le nom de l'objet qu'il venait de représenter. D’autre part, afin de l'habituer à écouter et d'améliorer sa compréhension, j'utilisais des périphrases au lieu de nommer les choses de manière abrupte. » (3).

La seconde phase de rééducation consiste à inciter Sabadel à illustrer des faits d'actualité et à traduire par le dessin des histoires qu'il entend, à lire les journaux ou écouter la radio un maximum.

On peut déjà cerner les grands traits des principales compétences du soignant en suivant la thérapie de Van Eeckhout ici :

- Le thérapeute associe toujours en premier lieu son patient à la thérapie ne serait-ce que pour des raisons d'efficacité, chacun des acteurs est donc coresponsable de l'amélioration de l'état du patient (la responsabilité du patient qui, s'il en était privé, supprimerait par le même coup une partie de sa liberté).

- Le thérapeute est avant tout une personne qui fait tout pour écouter, observer finement les comportements de son patient et de ses proches (Van Eeckhout rebondit souvent sur une remarque qu'il a entendue à propos de Sabadel à l'extérieur et qui lui a inspiré une partie de sa thérapie à venir).

- Enfin le thérapeute met en jeu son travail sur un temps long : plusieurs fois l'orthophoniste mentionne des marqueurs temporels comme « après quelques mois ". On voit que la thérapie s'inscrit sur du long terme et qu'en attendant il faudra affronter ce qui peut apparaître comme des échecs répétés.

Le bon thérapeute, c'est donc celui qui sait prendre des risques sans attendre de certitude quant à l'efficacité de son traitement, il est donc prêt à laisser se déployer une certaine créativité : « Cela supposait de notre part une foi et une 
persévérance qui font penser au pari de Pascal. Nous allions de découvertes en découvertes $॥$. (3)

III. Après cette présentation de l'aphasie et d'un vécu, je m'intéresse à l'infirmité motrice cérébrale (IMC),. La différence avec mon premier cas d'étude est principalement liée dans les cas d'IMC à la période de survenue du traumatisme, anté- ou périnatale ici par définition. Je m'intéresserai davantage dans ce second cas aux stratégies que peuvent déployer les personnes atteintes d'IMC pour trouver d'autres moyens de communiquer avec le monde, alors que pour les personnes aphasiques j'ai attaché plus d'importance à l'expérience vécue de la difficulté à vivre le handicap.

\section{Deuxième cas d'étude : les personnes IMC}

L'IMC survient dans la phase anté- ou périnatale et peut donc avoir de graves conséquences sur le cerveau de l'enfant en bas âge qui est encore en maturation, d'où des problèmes ultérieurs qui peuvent émerger. Les symptômes les plus fréquents résident dans des troubles de la posture et du mouvement, parfois de l'articulation qui fragilise l'élocution mais ne sont pas toujours présents. Là aussi, on observe un grand nombre de cas cliniques donc des prises en charge à adapter chaque fois au cas par cas. Les facultés intellectuelles sont suffisamment préservées pour permettre une scolarisation relativement normale, à la différence des IMOC (infirmité motrice d’origine cérébrale) dont la déficience intellectuelle gêne encore le développement du jeune enfant.

La participation sociale est encore plus lésée lorsqu'il y a des troubles de l'articulation. L'individu ne peut pas accéder à la même qualité de service en termes de réseaux de santé notamment. Les individus se retrouvent souvent en détresse émotionnelle, isolés socialement, renvoyés à une image très négative d'eux-mêmes. ${ }^{5}$ Pour pallier à cette limitation de la participation sociale, je développe deux points : les outils mis en place de 
façon individuelle ou standardisée pour améliorer la communication alternative (CAA), mais aussi d'une manière plus adaptée à chaque cas, l'émergence d'une relation de binôme entre la personne aidée et la personne qui la retranscrit et lui permet de s'exprimer en public :ceux qu'on promeut ici sous le terme d'assistants de communication bien que ce ne soit pas encore un métier reconnu aujourd'hui.

La communication alternative améliorée vise donc à soutenir, à compléter le langage parmi les IMC. Les premiers outils apparaissent au Canada après l'adaptation en 1971 du système que Karl Blitz développe pendant la Seconde Guerre mondiale pour élaborer un langage international, il s'agit du système Bliss (Bliss Scanography 4500 symboles traduits en plus de 25 langues). En Angleterre en 1975, on assiste à la création du système Makaton (association de pictogrammes et signes). En 1983, I'association internationale ISAAC naît au Canada (aujourd'hui elle compte 3000 membres dans 60 pays), elle permet la diffusion d'informations et des échanges parmi les personnes concernées, les familles, les professionnels et experts sur les thématiques de la CAA qu'elle vise à faire reconnaître.

Louis Avan (Arts et Métiers) et Danièle Truscelli (hôpital du Kremlin-Bicètre), en 1975, commencent une collecte de données sur les situations de communication en fonction des partenaires en présence. Dans les années 80 , on assiste à une multiplication de méthodes " officielles ", de banques d'images et d'outils spécifiques à certaines populations. Mais le grand tournant survient entre les années 80 et 90 avec l'essor de l'informatique et d'internet.

Dès 1975, apparaissent des logiciels et des matériels donnant accès à l'écriture à ceux qui ne peuvent pas se servir de machines à écrire : par exemple la machine Carba et la commande à la langue Carba Linguaduc (conçue par Claude Gabus). Les débuts de la voix artificielle apparaissent quant à eux en 1982 avec la synthèse vocale Sparte de France 
Télecom. En 1984, c'est l'apparition des premiers appareils dédiés à la CAA (lettres sous formes phonétiques). Un appareil est conçu pour les utilisateurs du Bliss : Mutavox (système symboliques de 384 cases). La démocratisation de l'informatique et la multiplication des outils technologiques ont donc un impact énorme sur l'efficacité de l'expression et l'autonomie des personnes.

Les méthodes d'encodage de la CAA sont très porteuses, mais avant tout, il faut connaître le code utilisé afin de comprendre la personne qui en a besoin ce qui reste fondamentalement l'apanage d'un cercle restreint. Les solutions à ces difficultés sont complétées par l'assistant de communication qui est toujours le fruit d’un binôme adapté au cas par cas entre les deux parties. Sans cette aide, les témoignages des personnes concernées rapportent que l'interlocuteur, surtout quand il ne connaît pas encore la personne qui a du mal à s'exprimer se sent gêné, évite d'échanger avec cette dernière, parle à la personne qui l'accompagne sans s'adresser directement à elle. Aux yeux de leurs interlocuteurs, elles apparaissent souvent comme incompétentes mentalement dès lors que leurs problèmes d'élocution se manifestent, et elles sont infantilisées (ce qui est notablement aggravé lorsqu'elles sont également en fauteuil manuel ou électrique). L'interlocuteur ne laisse pas le temps à la personne de s'exprimer ; plus largement, il ne lui permet pas d'avoir un environnement qui va lui permettre d'exprimer ses capacités. S'ensuit un désarroi réciproque qui entraîne une crainte de la rencontre des personnes en difficulté, une limitation de la participation sociale qui peut alors être palliée par un assistant de communication.

Ce nouveau métier existe déjà au Canada et en Suède mais n'est pas encore institutionnalisé en France. II soulève de nombreuses questions, par exemple quant au financement du service, assistance indépendante du métier d'auxiliaire de vie (la distinction 
est importante car les auxiliaires jouent des rôles complètement différents qui sollicitent des compétences inassimilables auprès de la personne concernée ; par exemple pour être assistant de communication il faut à la fois être capable de mobiliser des ressources attentionnelles tout en ayant un bagage universitaire fourni, alors que l'auxiliaire de vie mobilise plutôt des compétences physiques et techniques), au choix de l'assistant de communication par la personne concernée et en fonction de ses besoins (et non pas en fonction des disponibilités du service), à l'adéquation de l'assistant de communication en fonction du sujet ou des mots à traduire, avec des connaissances parfois nécessaires du sujet traité. Et bien sûr, une formation spécifique par rapport à celle de l'auxiliaire de vie.

Le métier d'assistant de communication est très délicat : c'est le traducteur, pas l'interlocuteur. II se doit de garder une fidélité mot pour mot au message transmis, de se positionner en retrait et savoir rester discret. II aide la personne qu'il assiste comme elle le lui demande pour la prise de parole et l'expression. D'une manière générale, l'assistant de communication renforce et augmente la qualité des contacts entre les partenaires (il a une place privilégiée par rapport à de simples bénévoles ou aux familles qui sont souvent trop impliquées).

Stéphane Irigoyen, conseiller départemental à l'APF, témoigne de sa qualité de vie accrue lorsqu'il a pu bénéficier de l'aide de son assistant de communication :

Le fait d'être assisté pour ma communication dans toutes mes actions de conseiller départemental de manière systématique a changé presque tout dans mon militantisme. Tout d'abord, je ne l'avais pas anticipé, cette aide m'apporte la constance dans mes prises de parole. Au problème d'élocution s'ajoute les conséquences de l'instabilité, pas toujours perçue, y compris par moi-même, de mon Infirmité Motrice Cérébrale (contractions, facilité pour parler, pour se déplacer...) 
...Certains jours je pouvais rester sans dire un mot : trop d'efforts. Avec mon assistant de communication, j’arrive à surmonter cette léthargie car j’ai beaucoup moins d'efforts à faire pour être compris. Au tout début, j'ai eu peur que l'assistant de communication soit un recul par rapport au travail que j'effectue avec mon orthophoniste pour justement m'exprimer oralement. Mais au bout de quelques semaines j'ai vite compris la différence que cette aide m'apportait dans mes relations aux autres. Les autres se sentent beaucoup plus à l'aise... Je ne suis plus obligé de dire uniquement l'essentiel, je peux développer mes idées, raconter une anecdote, faire des apartés, bref être comme la plupart des gens qui parlent sans entraves... Je peux arriver à un rendez-vous « les mains dans les poches " 6 Philippe Aubert, IMC de 41 ans, chercheur en sociologie, est quant à lui totalement dans l'incapacité de prendre tout seul la parole même si une fois aidé ou aux commandes de son ordinateur muni de capteurs visuels, il peut s'exprimer et interagir de façon tout à fait ordinaire avec le monde qui l'entoure.

II nous livre un témoignage dans son autobiographie La Rage d'exister. II explique ses besoins particuliers en termes de communication où il attend beaucoup d'écoute et d'attention de son interlocuteur : «Pour que nous entrions dans un dialogue, mon interlocuteur doit savoir doser l'écoute et l'interprétation, attendre de deviner ma pensée de façon presque certaine avant de $\mathrm{m}^{\prime}$ en proposer une formulation. " $(25)^{7}$. II est évident que communiquer avec lui prend beaucoup plus de temps qu'à l'ordinaire comme il le souligne :

Une lettre = 15 secondes ; une ligne de 75 lettres = 20 minutes ; une page de 40 lignes $=13$ heures. Cette estimation n'a rien d'exagéré $:$ c'est bel et bien le temps 
dont j'ai besoin avec un interlocuteur novice. L'exercice est un peu plus rapide une fois que nous sommes habitués l'un à l'autre. (45)

Pour que la communication soit réussie, il insiste sur l'importance du suivi pour la personne concernée d'autant qu'une mauvaise communication est un échec que partage aussi

l'aidant :

Imaginez combien il est angoissant d'être livré à quelqu'un qui, de prime abord, ne vous comprend tout simplement pas. Imaginez aussi comment il est perturbant pour un professionnel d'être en charge d'une personne avec laquelle elle ne parvient pas à échanger. (52)

A nouveau se déploie la difficulté de la tâche d'assistant qui doit savoir bien doser son aide ni trop généreuse ni trop limitée pour aider véritablement la personne assistée : «Toutefois, il [l'assistant de communication] doit lui-même se fixer des limites : à trop intervenir auprès de la personne handicapée, il entretient finalement sa dépendance. C'est, en fin de compte, une négociation permanente » (58).

\section{Troisième cas d'étude : les patients LIS}

Le syndrome d'enfermement (en anglais locked-in syndrome), connu aussi sous le nom de syndrome de verrouillage, est une condition neurologique résultant d'un accident. Le patient est éveillé et totalement conscient, mais il ne peut ni bouger ni parler en raison d'une paralysie complète, excepté pour le mouvement des paupières et parfois des yeux. Toutefois ses facultés cognitives restent intactes. Le LIS est souvent causé par un AVC (plus rarement par un traumatisme du pont qui est une partie du tronc cérébral). Le terme « locked-in » est dérivé de l'expression anglaise signifiant « verrouillé de l'intérieur », ce qui traduit bien le tableau clinique typique de ce syndrome : un état de pleine conscience néanmoins associé à une paralysie quasi-totale de l’organisme. Les premiers patients atteints du LIS sont déclarés en état de mort apparente. Le LIS était encore très peu étudié il y a quelques décennies. C'est grâce au livre Le Scaphandre et le 
Papillon de Jean-Dominique Bauby $(1997)^{8}$ et au film éponyme (2007) y que le syndrome est plus largement médiatisé. Dans cet ouvrage, Bauby métaphorise son état comme celui d’un scaphandrier qui ne voit le monde qu'à partir de son hublot, l'autre partie du titre « papillon » faisant oxymoriquement référence à la légèreté de la pensée qui l’accompagne. "Loin de ces raffuts, dans le silence reconquis je peux écouter les papillons qui volent à travers ma tête. II faut beaucoup d'attention et même du recueillement car leurs battements d'ailes sont presque imperceptibles. Une respiration un peu forte suffit à les couvrir. » (103).

En regard de cet ouvrage aux résonnances poétiques, on peut lire le compte-rendu plus terreà-terre d'un de ses thérapeutes, Van Eeckhout à l'aide du suivi avec lequel il accompagne JeanDominique Bauby. Van Eeckhout rencontre Bauby lorsque ce dernier a quitté l'hôpital parisien de Lariboisière pour se rendre à Berck : Philippe Van Eeckhout observe une respiration courte, une incapacité à déglutir, les principaux symptômes d'un Locked-in syndrom complet et irréversible. Le thérapeute se rend à Berck une fois par semaine, Van Eeckout reconnait le travail remarquable de sa collègue Sandrine auprès de Jean-Dominique Bauby qui lui permet d'ailleurs d'écrire son ouvrage, Le Scaphandre et le papillon. Pour cela, Jean-Dominique et Sandrine se mettent au point sur un code : « Ce code lui avait été rapidement enseigné par l’orthophoniste. II s'agit d'un alphabet où voyelles et consonnes sont ordonnancées suivant leur fréquence d'utilisation dans la langue française : E S A R I N T U L O M D P C F B U G J Q Z Y X K W. Jean-Dominique clignait de l'œil pour arrêter son interlocuteur sur la lettre qu'il devait prendre en note » (197).

L'autre patient qui a permis une médiatisation plus large de ce syndrome, est Philippe Vigand qui parle dans son premier ouvrage Putain de silence $(1997)^{9}$, co-écrit avec l'aide de sa femme Stéphane Vigand de son accident et surtout de sa sortie du coma. Ici les trois témoignages (Philippe Vigand, Stéphane sa femme, Philippe Van Eeckhout) s'éclairent les uns les autres.

Son accident date de 1990, alors qu'il se rend au bureau, il perd connaissance puis tombe dans le coma. Quand il se réveille, plus aucune partie de son corps ne répond en dehors du battement des paupières. 
Dans son quatrième livre Légume vert $(2011)^{10}$ Philippe Vigand parle de son quotidien et des adaptations qui lui permettent d'avoir une vie sereine, notamment ses auxiliaires de vie ou encore son ordinateur adapté, son contrôleur d'environnement. Son ordinateur lui a notamment permis de rédiger les quelques lignes qu'il a écrites : « Ma toile à moi étant un écran d’ordinateur, et le pinceau ma pupille, fixée par une caméra qui cherche sur un alphabet (la palette) les lettres dont elle a besoin. » (PS, p.17). Au niveau des codes qui lui permettent de communiquer avec un tiers sans faire intervenir d'outil numérique, il explique qu'il utilise un tableau :

« Comment décrire ces moments de frustration extrême où j'ai l'impression d'être une mouche enfermée entre quatre vitres contre lesquelles elle ne cesse de se cogner, cherchant en vain une porte de sortie? 》 (16).

Philippe Vigand rend également compte de l'importance de l'auxiliaire de vie dans son existence « C'est un métier fort délicat puisqu'il consiste à réagir et à intervenir sans outrepasser son rôle de go-between, à s'intégrer dans la famille sans être pesant, à adapter son rythme et ses activités tout en sachant garder du recul, tout en conservant sa personnalité. » (PS, p. 67). Mais c'est surtout la durée qui confère une très grande familiarité et expertise à la personne qui l'accompagne sur le long terme, comme il en témoigne à propos d’Emmanuel : « Surtout, le fruit de cinq années d'accompagnement ont donné à Emmanuel un sens quasi divinatoire des propos que je veux tenir » (PS, p. 68). Voici ce qu'écrit Philippe : « ces précieux auxiliaires de vie font plus que remplacer mes bras et mes jambes immobiles : ils sont les acteurs de ma réintégration dans une existence presque normale. » (PS, p. 69), " qu'un auxiliaire de vie vienne à manquer, et tout notre organisation vacille » (LV, p. 19).

Adapter le matériel informatique semble quasi impossible à Philippe Vigand peu après son accident, le problème qui se pose est celui de la paralysie quasi complète du corps et donc de I'absence de mouvements : «L'idée était de trouver un outil de communication compatible avec mes 
mouvements possibles, c'est-à-dire aucun sauf celui des paupières. On choisit de ne pas chercher à reproduire ce qui existait déjà » (PS, p. 37). Il faut donc innover sans tenter de plaquer du déjà connu. Philippe Vigand parvient à nouveau à communiquer grâce à une caméra infrarouge qui détecte ses mouvements oculaires.

Philippe n'a de cesse d'insister sur le bien-être que lui confère son ordinateur à travers la possibilité qu'il lui donne de communiquer : « On entend souvent dire que le malheur vous apprend à vous détacher des choses matérielles pour mieux vous consacrer à l'essentiel. Sauf que, dans la vie d'un handicapé, tout ce qui est matériel se révèle absolument essentiel. Dans ma vie à moi, il est un objet dont je ne me passerais pour rien au monde : mon ordinateur. C'est au printemps 1991, un peu moins d'un an après mon accident, que cette merveille de technologie est entrée dans mon existence. » (LV, p. 20) et « Je n'enquiquine personne au téléphone en appelant plusieurs fois par jour, mais j'écris. A ma femme, à mes enfants, à mes amis » ( $L V$, p. 21$)$.

Son épouse, Stéphane Vigand, explique bien combien la notion du temps n'est pas la même dans les relations sociales avec son mari : « Là où il me faut trois secondes pour dire « passe-moi le bouquin qu'Emmanuel a laissé sur la table ", il en faut trois aussi à Philippe pour le penser, mais cinq minutes pour se faire comprendre ! Pour gagner du temps, la tentation est grande de finir ses phrases aves nos mots à nous, en nous persuadant que cela n'a aucune importance, l'essentiel étant de saisir le sens de ce qu'il veut dire. Mais, comme chacun de nous, Philippe a besoin de ses mots, sinon aucune véritable communication n'est possible. » (PS, p.135). Elle conclut : « Toute conversation digne de ce nom est une entreprise de longue haleine qui doit se faire dans le calme. » (PS, p.135).

Elle confirme cependant que l'écriture via l'ordinateur a permis à Philippe de s'exprimer beaucoup plus librement : «A travers l'écriture, je retrouve Philippe beaucoup mieux qu'entre les mots hachés du code. II n'avait jamais été un bavard impénitent ni un scribouillard de romansfleuves, son accident et la nécessité d'aller à l'essentiel l'ont rendu "synthétique de synthétique ». 
Au diable les fioritures et les circonvolutions ! Vive la concision! »(PS, p.138-139), « En tout cas, grâce à l'ordinateur, Philippe a retrouvé une vraie parole. Impossible de prétendre que l'on n'entend pas ses mots silencieux. » (PS , p.140)

\section{Conclusion}

L'aiguillon d'une communication empêchée est un moteur déterminant pour beaucoup d'entre nous à recréer de nouvelles façons d'échanger avec son entourage. A ce titre, la personne concernée ou son entourage, ou d'une manière plus générale celui qui œuvre à rétablir cette communication perdue avec toute la difficulté et le défi qu'implique cet engagement, " participe du processus de la création [de] formes nouvelles » (Flusser 1).

S'il s'agit toujours dans les différentes situations que nous décrivons de mettre en avant un aspect médical, c'est-à-dire ce que l'on observe de l'extérieur, le véritable cœur du problème reste la prise en compte de l'expérience vécue du sujet et la façon dont ce dernier s'approprie sa réalité. Ce n'est qu'à partir de cette seconde modalité que peuvent s'organiser des stratégies efficaces pour améliorer sa situation, en faisant intervenir l'ensemble des acteurs de son environnement, tel l'assistant de communication ou les pourvoyeurs de codes plus ou moins standards, plus ou moins adaptés sur mesure entre les interlocuteurs. Ces situations réinterrogent chaque fois l'aspect créateur de la démarche de l'individu et de son entourage qu'il soit parfois teinté de professionnels du monde du soin, démarche créative dont chacun doit garder en tête un vif impératif.

\section{Notes}

1. Vilem Flusser, «Le phénomène surprenant de la communication » Communication et langages, $n^{\circ} 37$, 1er trimestre 1978, 27-32.

2. Anne-Lyse Chabert, Transformer le handicap, Eres, Paris, 2017.

3. Philippe Van Eeckhout, Le Langage Blessé, Albin Michel, 2001. 
4. Sabadel, Une plume à mon cerveau, Editions Fabert, 2008.

5. Elizabeth Cataix-Nègre, Communiquer autrement, De Boeck Supérieur, 2017.

6. Stéphane Irigoyen cité par Elizabeth Cataix-Nègre, Communiquer autrement, De Boeck Supérieur, 2017.

7. Philippe Aubert et Sophie Jacolin, Rage d'exister, HD ateliers Henry Dougier, 2018.

8. Jean-Dominique Bauby, Le Scaphandre et le papillon, Editions Robert Laffont, Paris, 1997.

9. Philippe et Stéphane Vigand, Putain de silence, Editions Anne Carrière, Paris, 1997.

10. Philippe Vigand, Légume vert, Editions Anne Carrière, Paris 2011.

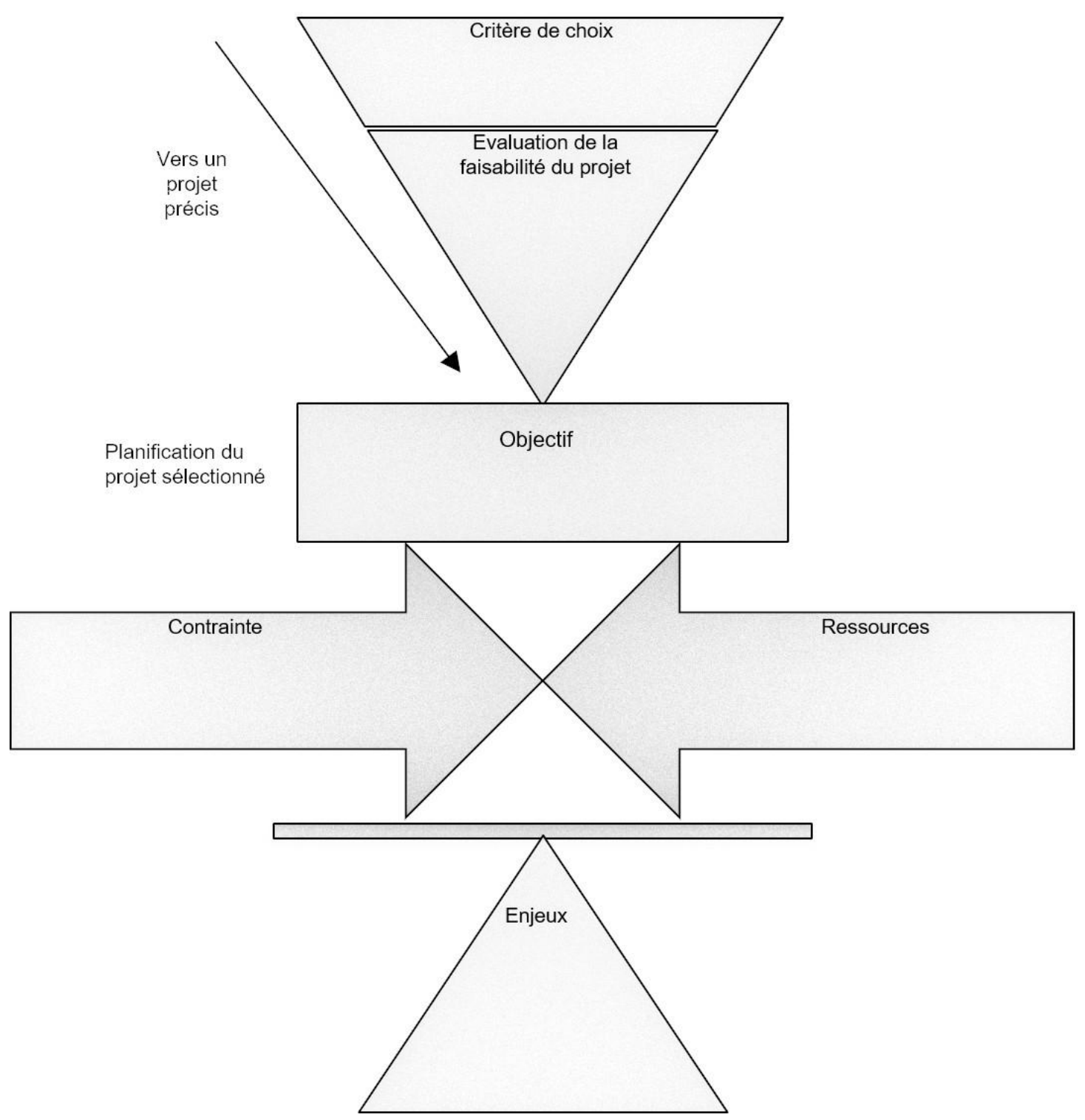

Schéma 1 - Paramètres à prendre en compte pour élaborer une solution 
1. Désordre initial de

l'individu inadapté à son

milieu (régulation

impossible)
2. Création d'un nouveau

cadre permettant la

régulation à l'individu

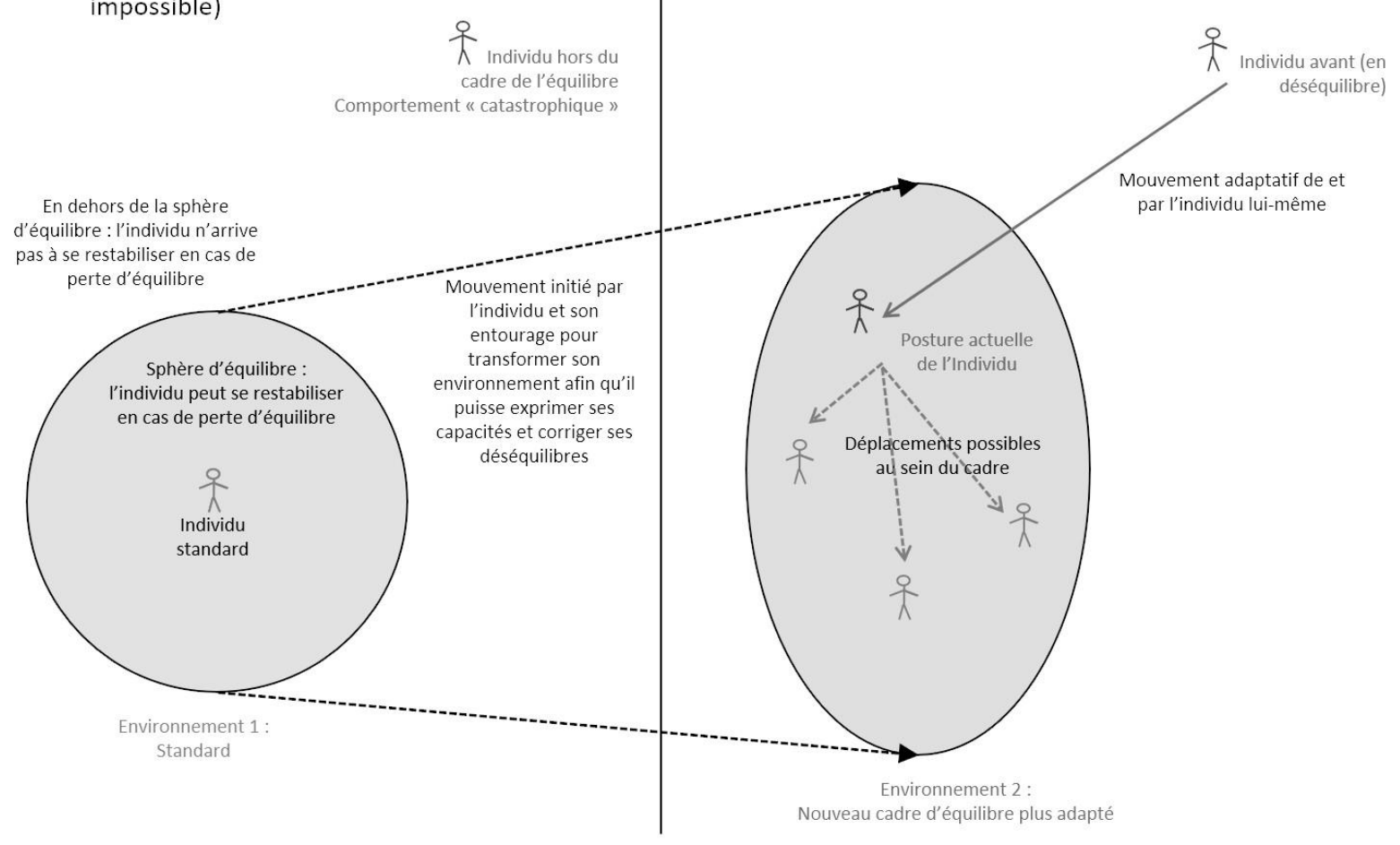

Schéma 2 - Double déplacement de l'individu et du milieu qui se coadaptent l'un à l'autre 


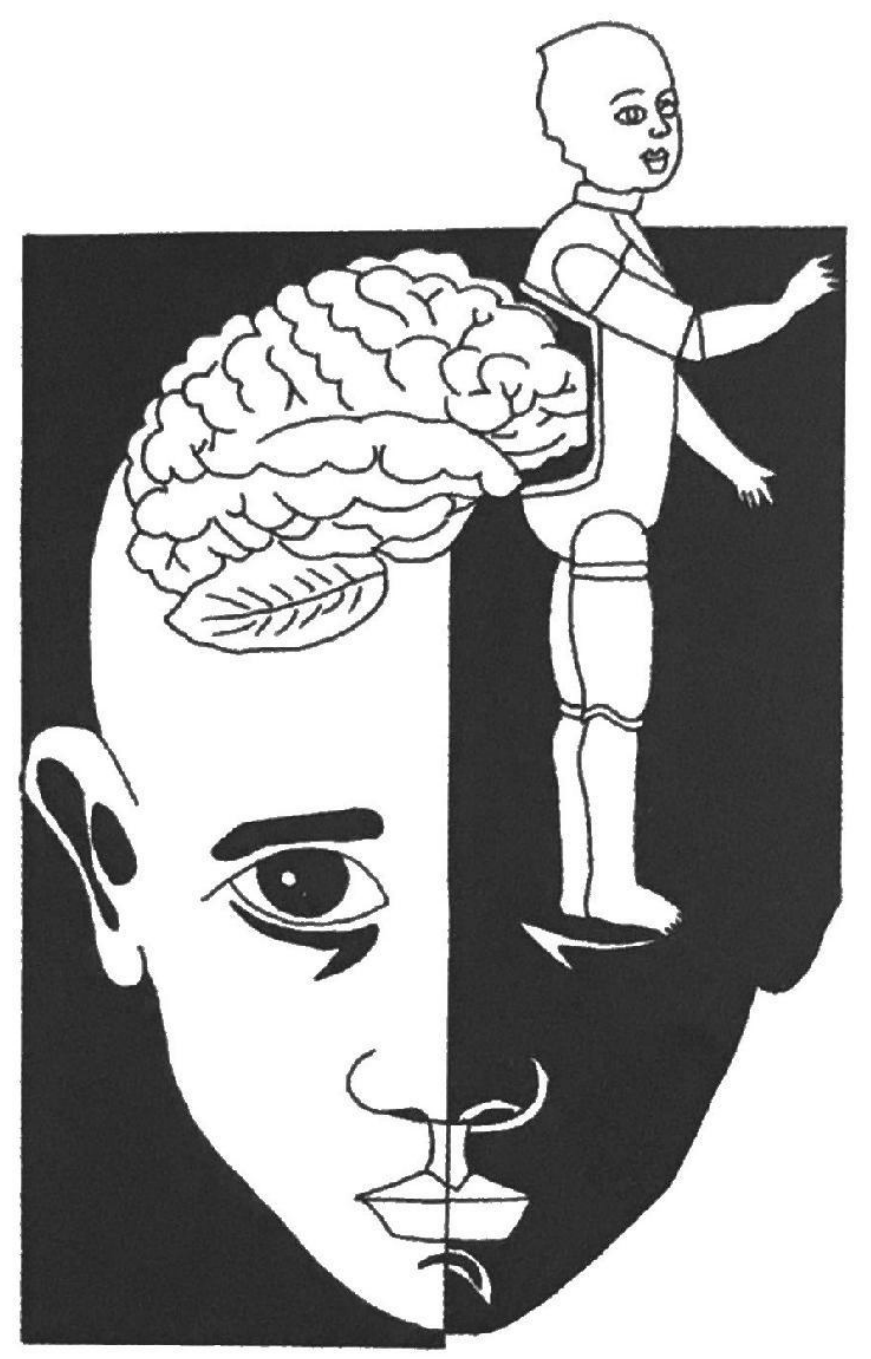

Dessin 1 - Page 34 


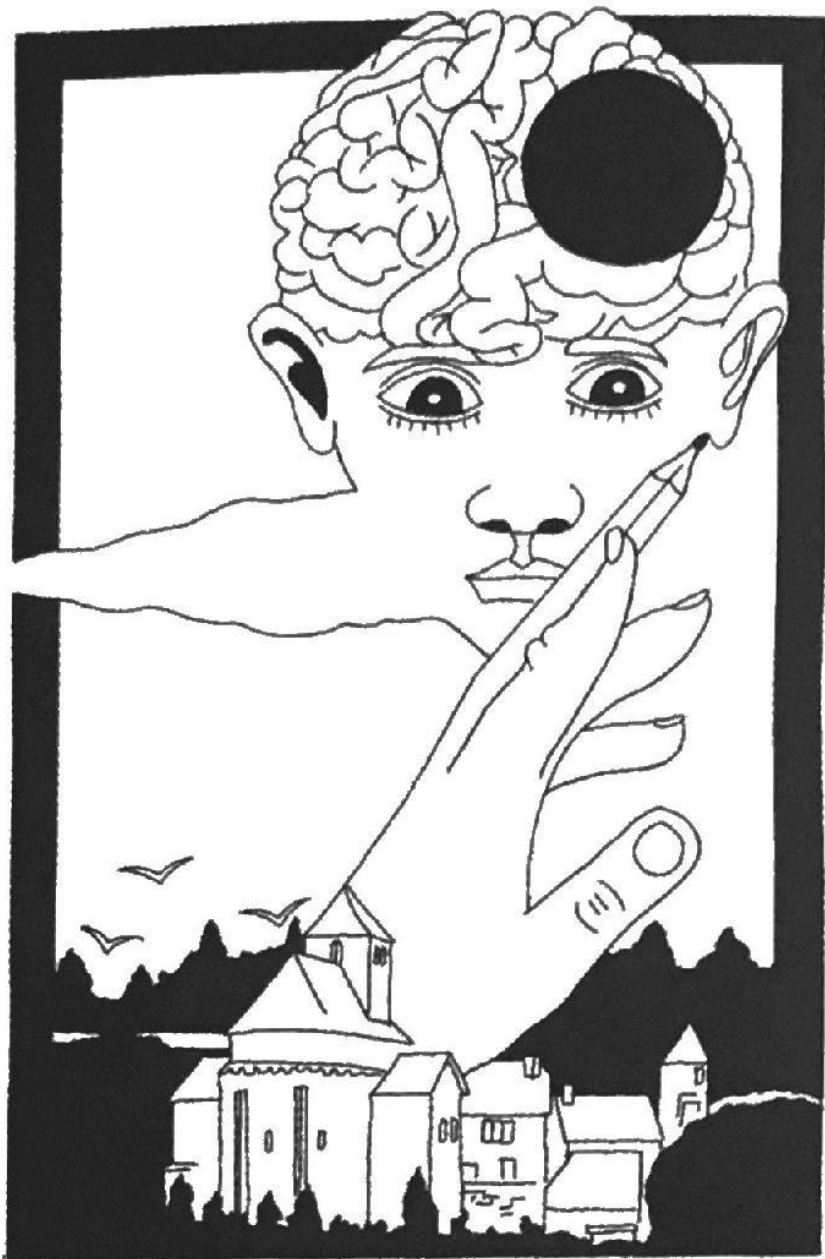

Dessin 2 - Page 56 


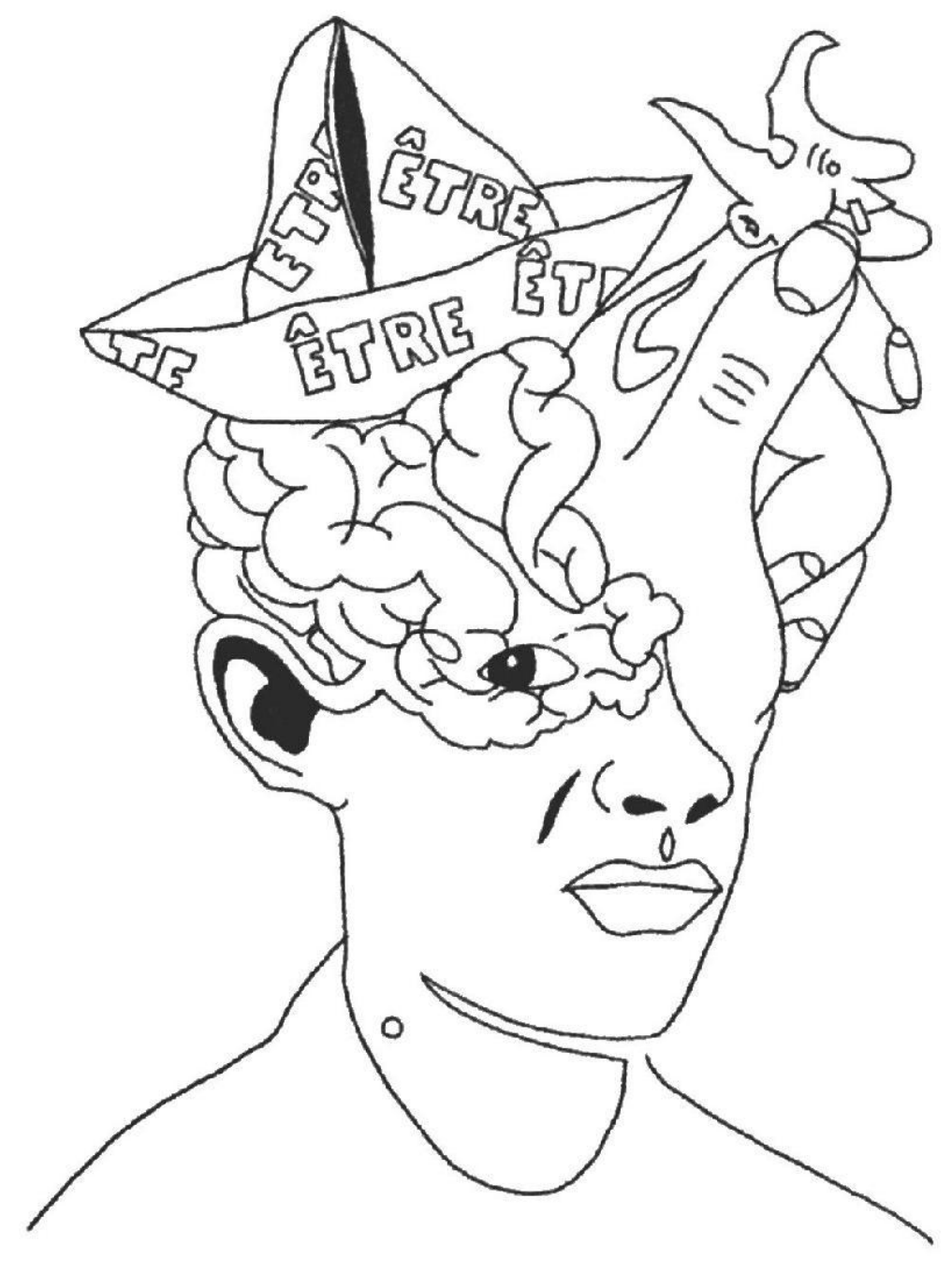

Dessin 3 - Page 96 